\title{
The successful arthroscopic treatment of suprascapular intraneural ganglion cysts
}

\author{
Nikhil K. Prasad, MBChB, ${ }^{4}$ Robert J. Spinner, MD, ${ }^{1,4}$ Jay Smith, MD, ${ }^{2}$ Benjamin M. Howe, MD, ${ }^{3}$ \\ Kimberly K. Amrami, MD, ${ }^{3,4}$ Joseph P. lannotti, MD, PhD, ${ }^{5}$ and Diane L. Dahm, MD1 \\ Departments of ${ }^{1}$ Orthopedics, ${ }^{2}$ Physical Medicine, ${ }^{3}$ Radiology, and ${ }^{4}$ Neurosurgery, Mayo Clinic, Rochester, Minnesota; and \\ ${ }^{5}$ Department of Orthopedics, Cleveland Clinic, Cleveland, Ohio
}

\begin{abstract}
OBJECT High-resolution magnetic resonance imaging (MRI) can distinguish between intraneural ganglion cysts and paralabral (extraneural) cysts at the glenohumeral joint. Suprascapular intraneural ganglion cysts share the same pathomechanism as their paralabral counterparts, emanating from a tear in the glenoid labrum. The authors present 2 cases to demonstrate that the identification and arthroscopic repair of labral tears form the cornerstone of treatment for intraneural ganglion cysts of the suprascapular nerve.
\end{abstract}

METHODS Two patients with suprascapular intraneural ganglion cysts were identified: 1 was recognized and treated prospectively, and the other, previously reported as a paralabral cyst, was identified retrospectively through the reinterpretation of high-resolution MR images.

RESULTS Both patients achieved full functional recovery and had complete radiological involution of the intraneural ganglion cysts at the 3-month and 12-month follow-ups, respectively.

CONCLUSIONS Previous reports of suprascapular intraneural ganglion cysts described treatment by an open approach to decompress the cysts and resect the articular nerve branch to the glenohumeral joint. The 2 cases in this report demonstrate that intraneural ganglion cysts, similar to paralabral cysts, can be treated with arthroscopic repair of the glenoid labrum without resection of the articular branch. This approach minimizes surgical morbidity and directly addresses the primary etiology of intraneural and extraneural ganglion cysts.

http://thejns.org/doi/abs/10.3171/2015.6.FOCUS15201

KEY WORDS intraneural ganglion cyst; labral tear; shoulder; unified articular (synovial) theory; suprascapular nerve; peripheral nerve imaging

$\mathrm{S}$ UPRASCAPULAR neuropathy has been recognized as a potential cause of shoulder pain and weakness. ${ }^{2,5}$ Along its course to innervate the supraspinatus muscle, infraspinatus muscle, and glenohumeral joint, the suprascapular nerve (SSN) passes through 2 potential points of impingement: the suprascapular notch and the spinoglenoid notch. ${ }^{41}$ Paralabral (extraneural) cysts are globular masses filled with mucoid material that emanate from the glenohumeral joint and can occasionally extrinsically compress the SSN at these anatomical bottlenecks., ${ }^{2,5} \mathrm{~A}$ rare variant form, the intraneural ganglion cyst, can extend within the SSN from the glenohumeral joint and assume a characteristic tubular pattern when assessed by MRI. ${ }^{30}$

Over the past 2 decades, the arthroscopic treatment of

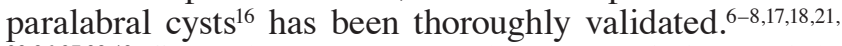
$23,26,27,39,40$ Several authors have shown that it is possible to treat this condition by repairing the coexistent labral pathology, without the need to perform an open or arthroscopic decompression or a resection of the cyst. ${ }^{23,26,40}$ Previously, an open approach was employed to treat intraneural ganglion cysts of the SSN by resecting the articular branch and decompressing or resecting the cyst. ${ }^{20,25,29,31,37}$

ABBREVIATIONS EMG = electromyography; FSE = fast spin echo; IS = infraspinatus; SLAP = superior labrum anteroposterior; SSN = suprascapular nerve; STIR = shorttau inversion recovery. 
Given the unifying articular theory, ${ }^{32}$ we predicted that it should be possible to treat suprascapular intraneural ganglion cysts in the same way as paralabral cysts - with repair of the capsulolabral defect in the joint of origin. Here, we present 2 cases of suprascapular intraneural ganglion cysts, scrutinized with high-resolution MRI and treated with arthroscopic repair of an associated paralabral tear, without resection of the articular branch.

\section{Illustrative Cases}

All retrospective reviewing of patient data was performed under the auspices of the institutional review board at the Mayo Clinic. Both cases were hypothesis driven. In Case 1, an arthroscopic approach was employed for the first time for a suprascapular intraneural cyst based on its analogous nature to a paralabral cyst. Case 2, which had been documented ${ }^{13}$ (and treated) as a paralabral cyst, had the MRI appearance of and was subsequently reinterpreted as an intraneural ganglion cyst.

\section{Case 1}

\section{History and Examination}

A 38-year-old woman presented with a 2-week history of gradually worsening, atraumatic, right posterior shoulder pain radiating down the arm and associated with paresthesia in the palm. Examination revealed mild denervation atrophy and weakness in the infraspinatus and supraspinatus muscles with preserved function in the deltoid, triceps, wrist and finger extensors, and latissimus dorsi muscles. She had mildly reduced sensation in the volar forearm, and the Tinel sign was positive in the infraclavicular region, radiating to the forearm. Electrodiagnostic studies were consistent with a suprascapular neuropathy proximal to the suprascapular notch, with denervation, reinnervation, and fibrillations in the infraspinatus and supraspinatus muscles. There were no electromyography (EMG) abnormalities noted in the ipsilateral deltoid or biceps muscles.
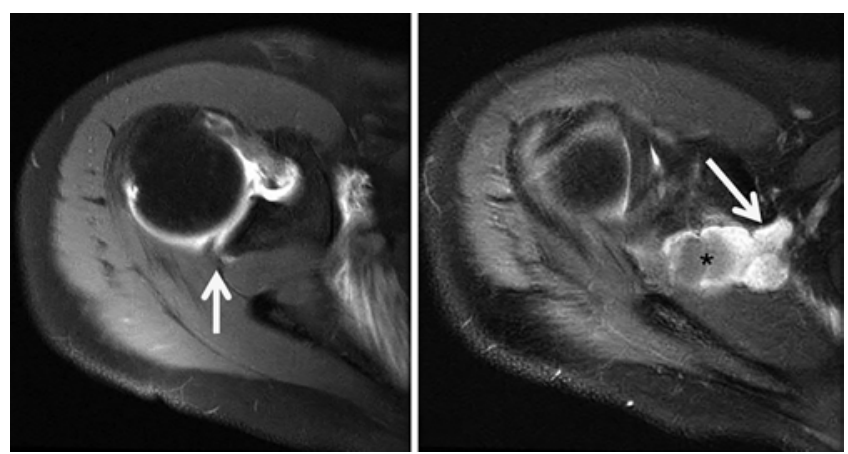

FIG. 1. Case 1. Preoperative MR images. Left: Axial T1-weighted fast spin echo (FSE) MR image with fat suppression after the administration of intraarticular gadolinium contrast agent demonstrates a tear in the posterior glenoid labrum with a connection to the paralabral cyst (arrow). Right: Axial T2-weighted FSE MR image with fat suppression after intraarticular gadolinium administration shows an intraneural cyst (asterisk) arising from the posterior labral tear (not shown) at the shoulder extending through the SSN into the upper trunk of the brachial plexus (arrow).
The MR image, which was previously described by our group ${ }^{33}$ revealed a labral tear posterior to the biceps labral complex with a well-defined narrow neck emanating from the tear (Fig. 1 left). The cyst traveled along the course of the SSN through the spinoglenoid and suprascapular notches, toward the upper trunk of the brachial plexus. The supraspinatus and infraspinatus muscles exhibited early signs of denervation with intramuscular edema but no atrophy. All other C5- and C6-innervated muscles within the radiological field of view appeared normal. Shoulder MR arthrography demonstrated intraarticular contrast extending from the labral tear as a narrow neck into the intraneural cyst (Fig. 1 right). Preoperative ultrasound confirmed these findings and provided a baseline for comparison with subsequent assessments.

\section{Treatment}

The patient underwent an arthroscopic evaluation of the right shoulder (Fig. 2). Anterior and posterior arthroscopy portals were used, the former through direct visualization of the rotator interval. No articular surface pathology was identified, but there was extensive fraying of the superior labrum, with a tear along the posterosuperior labrum extending from the 10 to 12 o'clock position. A probe was placed beneath the labral tear to identify the cyst. As the posterosuperior glenoid was rasped in preparation for repair, a small amount of cyst fluid was noted to enter the joint. The labral tear was repaired with the placement of No. 2 sutures in a mattress formation, anchored to a Bio-SutureTak. The articular branch of the SSN was not resected.

\section{Posttreatment Course}

Two weeks after surgery, there was $50 \%$ resorption of the cyst as detected by ultrasonography (Fig. 3). By 3 months after surgery, MRI revealed complete resolution of the cyst (Fig. 4) and EMG demonstrated reinnervation. One year postoperatively, there was complete resolution of the cyst as measured by ultrasonography and MRI. The patient made a complete functional recovery.

\section{Case 2}

\section{History and Examination}

A 27-year-old woman was evaluated for right shoulder pain and a nontender mass in the supraclavicular fossa.

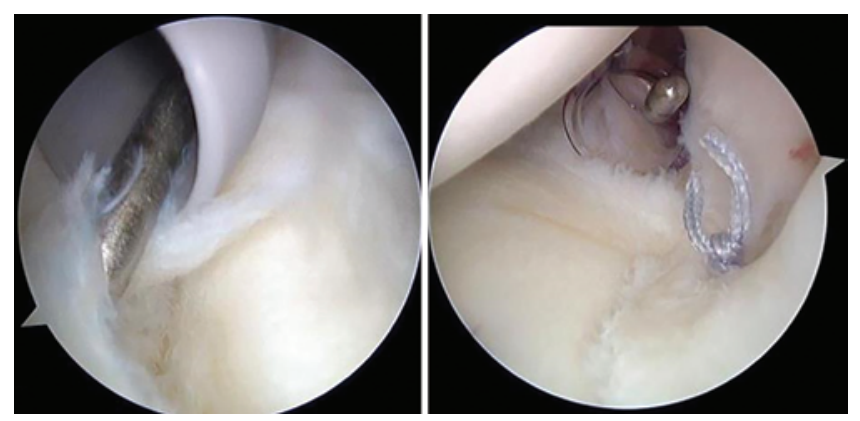

FIG. 2. Case 1. Intraoperative findings. Left: A posterosuperior labral tear viewed from the posterior portal. Right: Repair of the posterosuperior labral tear viewed from the anterior portal. 

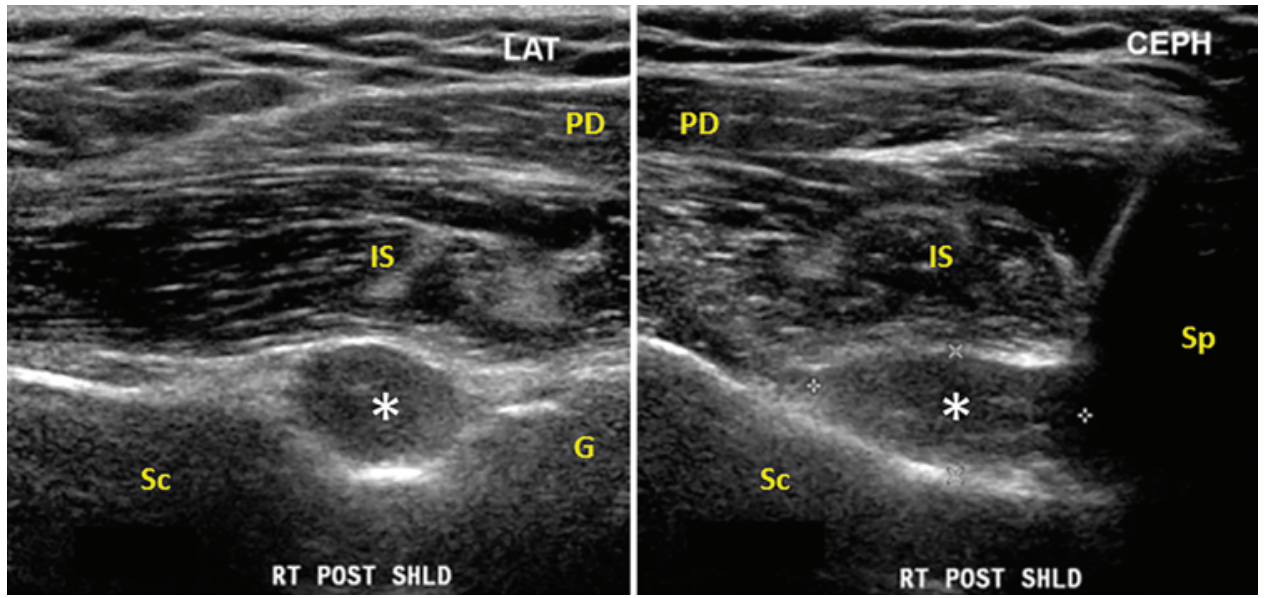

FIG. 3. Case 1. Ultrasound findings 2 weeks postoperatively. Left: Long-axis view of the infraspinatus muscle demonstrates persistence of the spinoglenoid notch cyst (asterisk). The relationship among the cyst, glenoid (G), and scapula (Sc) is clear. Orientation: left $=$ medial, right $=$ lateral $($ LAT), top = superficial, bottom = deep. Right: Short-axis view of the infraspinatus muscle demonstrates persistence of the spinoglenoid notch cyst (asterisk). The relationship among the spine of the scapula (Sp), infraspinatus fossa (Sc), infraspinatus (IS), and posterior deltoid (PD) is clear. Orientation: left = caudad, right $=$ cephalad $(C E P H)$, top = superficial, bottom $=$ deep .

The shoulder pain was exacerbated by overhead activities, but the patient's primary concern was the cosmetic appearance of the neck mass. There was no history of trauma, and the patient was not an athlete. Neurovascular function of the right upper limb was intact. MR images showed a ganglion cyst that was thought to be a paralabral cyst $^{13}$ (Fig. 5) extending from a torn edge of the superior labrum anteroposterior (SLAP) Type II to the supraclavicular fossa.

\section{Treatment}

A combined arthroscopic and open approach was used-the former to treat the labral tear and the latter to excise the subcutaneous portion of the cyst with an incision made directly over it. As the pathology was thought to be extraneural, no attempt was made to resect the articular nerve branch at the time of operation. The resected segment of cyst did not contain any neural tissue.

\section{Posttreatment Course}

No electrodiagnostic studies were performed before or after the operation. The patient achieved functional improvement secondary to relief of shoulder pain, and both gross and radiological involution of the cyst occurred by the 3-month follow-up (Fig. 6).

The cyst's morphology was intriguing ${ }^{19}$ and seemed reminiscent of an intraneural ganglion cyst, with a tubular proximal extension along the course of the SSN. With the assistance of the senior author, we were able to obtain all relevant clinical data and the original imaging. Threedimensional reconstructions of the images confirmed our suspicions that it was an intraneural ganglion cyst with a tubular morphology (Fig. 7A and B) and with evidence of crossover (in the upper trunk), descent (in the divisions), and ascent as far as the C-5 and C-6 nerves (Fig. 7C). The largest collection of cyst fluid appeared to be in a cutaneous branch of the SSN. There was subtle MRI evidence of denervation changes in the supraspinatus and infraspinatus muscles, i.e., increased signal on short-tau inversionrecovery (STIR) sequences, consistent with an occult neuropathy of the SSN.

\section{Discussion}

The 2 illustrated cases in this study represent an important milestone in our understanding of the pathophysiology and treatment of intraneural ganglia. The cases show that intraneural ganglion cysts of the shoulder share a common etiology with paralabral cysts and can be treated in a similar fashion. These findings are consistent with the unifying theory for intraneural ganglia, ${ }^{34}$ which explains the development of both intraneural and extraneural ganglion cysts (Fig. 8) and allows us to target treatment to the primum movens (the joint) and predict surgical outcome. While we acknowledge that cyst decompression, either ar-
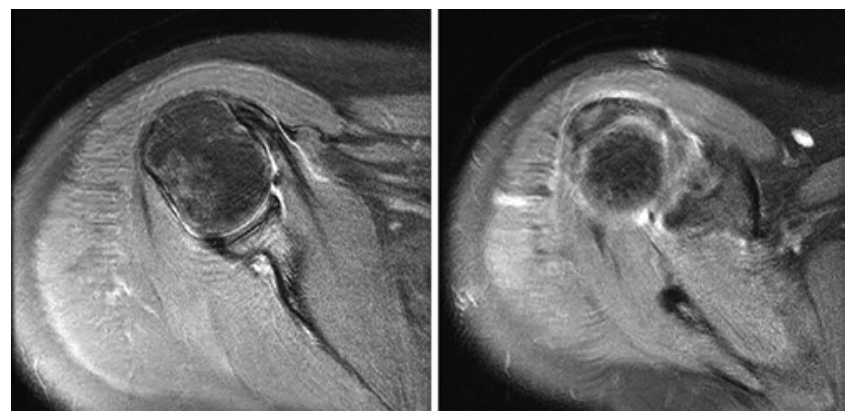

FIG. 4. Case 1. Postoperative MR images at 3 months after treatment. Left: Axial T2-weighted FSE MR image with fat suppression (same level as image seen in Fig. 1 left) after repair of the labral tear shows resolution of the tear and cyst. Right: Axial T2-weighted FSE MR image with fat suppression (same level as image seen in Fig. 1 right) after repair of the labrum shows complete resolution of the intraneural cyst, including extension into the brachial plexus. 

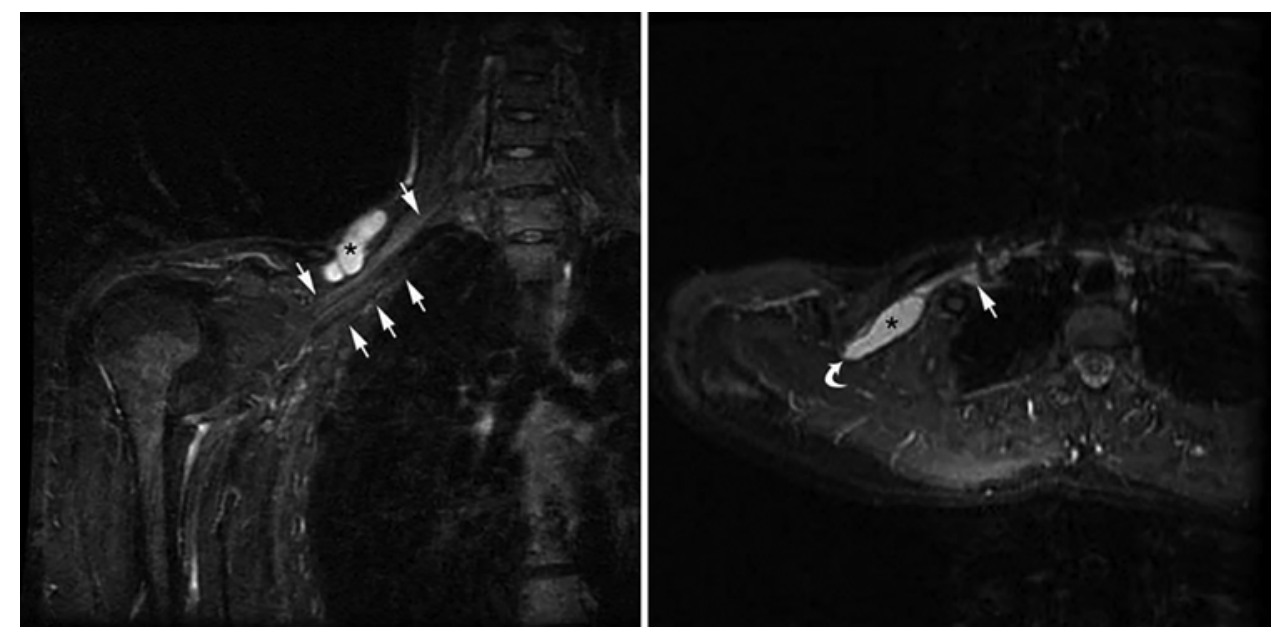

FIG. 5. Case 2. Preoperative MR images. Left: Coronal STIR MR image slightly anterior to the published image ${ }^{13}$ shows a large cyst (asterisk) within the soft tissues of the right neck extending toward the skin, seemingly remote from the shoulder joint. The slightly hyperintense brachial plexus (arrows) is immediately deep to the cyst. Right: Axial STIR MR image of the right upper chest shows the large cyst (asterisk) within the SSN with extension anteriorly deep to the clavicle. There is a subtle suggestion of the proximal extension of cyst to the cervical roots (straight arrow). The posterior aspect of the cyst (curved arrow) is just superior to the suprascapular notch.

throscopically (Case 1) or with an open approach (Case 2), may provide immediate relief of symptoms, we do not believe that it is necessary for long-term success of treatment and should not be regarded as the primary therapeutic objective.

The most widely accepted theory for the pathogenesis of ganglion cysts is that joint fluid egresses into the para-

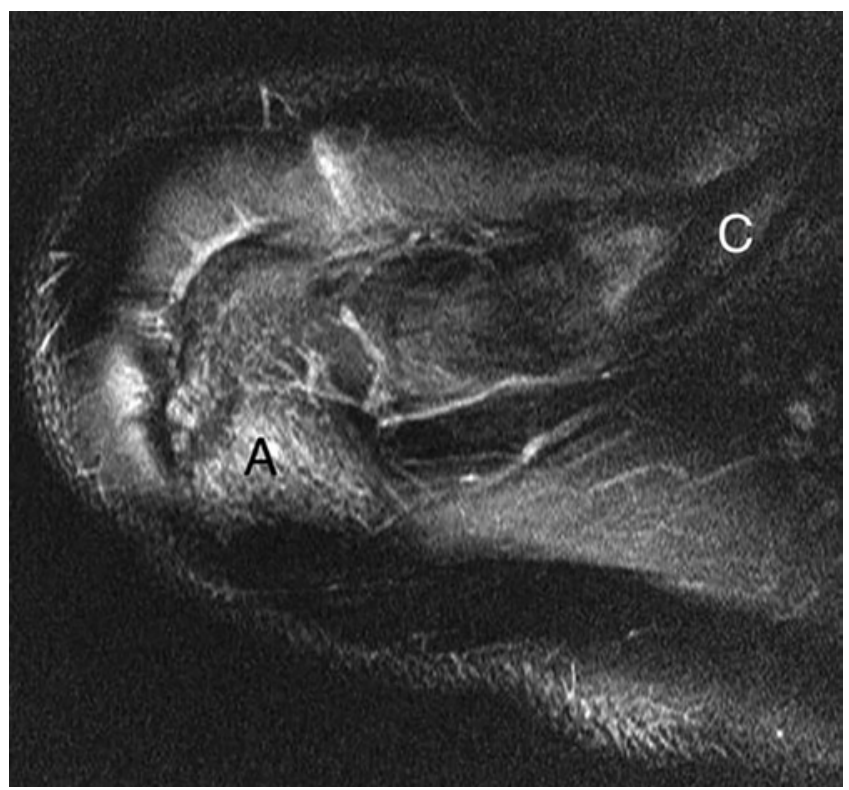

FIG. 6. Case 2. Postoperative MR image at 2 months after treatment. Axial T2-weighted FSE MR image with fat suppression at the level of the acromion ( $A$; same axial level as in Fig. 1 right) shows no cyst deep to the clavicle $(C)$ after repair of the labral tear and resection of the most superficial component of the cyst in the neck. The postoperative study is very limited, performed with a shoulder coil with a small field of view and few sequences. articular tissues from a capsulolabral tear. The strength of the association between labral tears and both paralabral

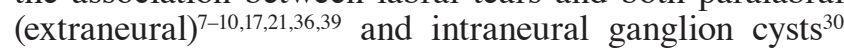
has been established. We have also previously demonstrated $^{30}$ and herein confirmed a similar association between posterosuperior labral tears and intraneural ganglion cysts. The intraneural ganglion cysts in Cases 1 and 2 obey the same principles of formation as previously reported suprascapular intraneural ganglia: $:^{20,22,24,25,29-31,37}$ 1) They originate from the articular nerve branch that supplies a synovial joint; 2) they follow the path of least resistance; and 3) the size, extension, and compressive effect of the cyst fluctuates based on dynamic joint pressures. These principles also apply to extraneural cysts, except that the joint connection is via a nonneural pedicle. In Case 1, the suprascapular intraneural ganglion cyst was identified preoperatively. ${ }^{33}$ The joint connection was established by MRI, and the communication through the labral tear was documented by MR arthrography. The cyst extended longitudinally toward the retroclavicular brachial plexus. Given our experience with this case and others, ${ }^{30,31,33}$ we were able to reinterpret the imaging findings of Case 2 . The cyst in Case 2 followed the same course as in Case 1, but it extended even more proximally along the brachial plexus (Fig. 7). We believe that the supraclavicular mass that was resected was actually a portion of the intraneural ganglion cyst tracking into a cutaneous branch of the SSN. The existence of this branch, though not widely known, has been demonstrated by several authors ${ }^{1,11,14,38}$ to consist of fine filaments that ramify with the lateral supraclavicular nerve. ${ }^{1}$ Given the small caliber of this twig and the large cyst, it is understandable that the nerve fascicle was not seen in the resected specimen (note that we have seen this in many cases of resected articular branches of peroneal intraneural ganglia). Alternatively, the subcutaneous cyst may represent extraneural rupture and cyst encapsulation. 

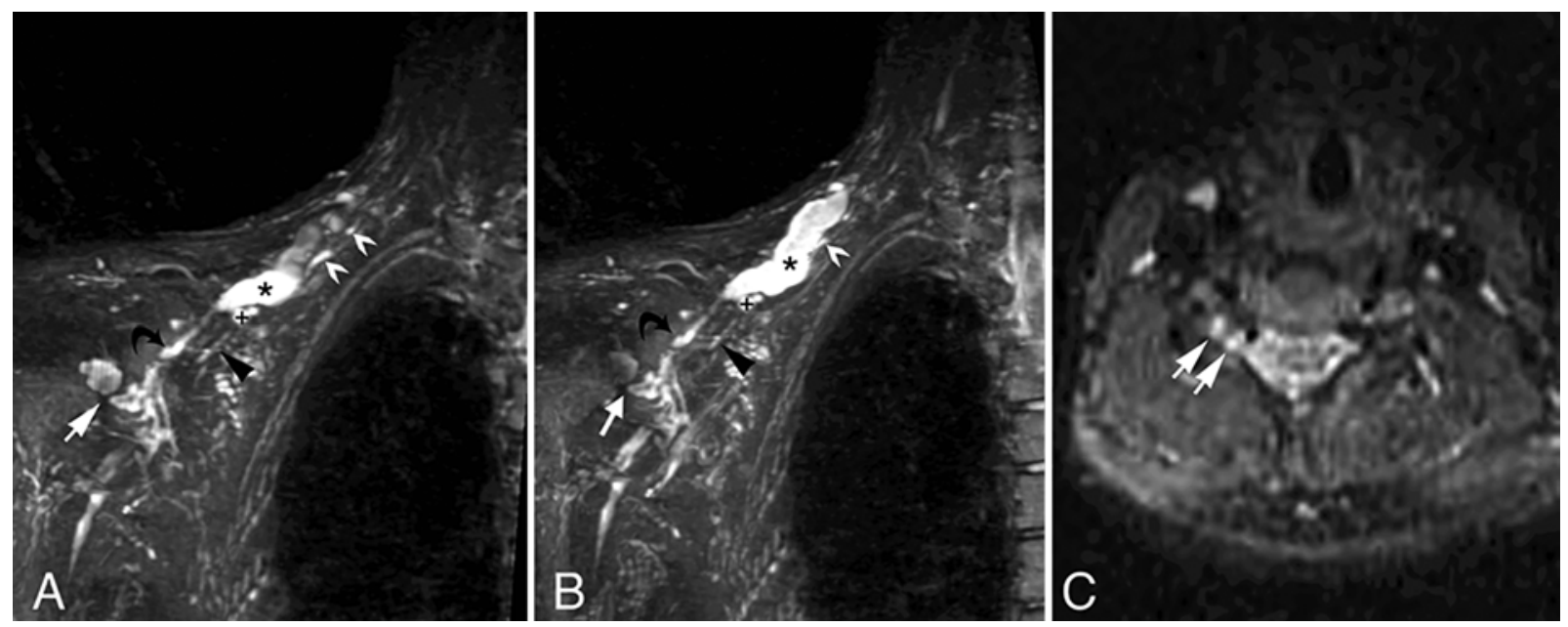

FIG. 7. Case 2. Reinterpreted preoperative MR images. A: Coronal maximum intensity projection (MIP) from a coronal STIR MR image data set shows the intraneural cyst (asterisk) arising from a labral tear at the shoulder (white arrow), extending within the SSN (black curved arrow) proximally into the neck. The image shows proximal extension of the cyst along C-6 (white arrowheads). The image also shows evidence of descent within the brachial plexus (black arrowhead) from a nexus point (black plus sign) at the inferior aspect of the larger cyst. B: Oblique coronal MIP from a coronal STIR MR image data set slightly anterior to the image in panel A shows the course of the cyst from the labral tear (white arrow), through the suprascapular cyst (black curved arrow) and into the larger cyst extending toward the skin (asterisk). There is again evidence for proximal extension along C-6 (white arrowhead) as well as the nexus point (black plus sign), intraneural ascent (white arrowhead), and descent (black arrowhead), as seen in panel A. C: Axial STIR MR image at the level of C-5 shows a cyst in the right nerve root within the foramen (arrows). The left side is normal.

It has been consistently demonstrated that treating labral pathology leads to cyst involution, regardless of whether the cyst is decompressed intraoperatively or not. ${ }^{23,26,40}$ However, decompression of paralabral cysts without addressing the labral tear often leads to recurrence. . $^{3,4,12,28,36,39}$ Given these findings, results from the operative treatment of SSN intraneural ganglia can also be predicted. In both of the reported cases, there was successful treatment of intraneural ganglia without resection of the articular nerve branches (intentionally in Case 1 and unknowingly in Case 2). The cyst decompression in Case 1 and the open resection of the subcutaneous portion of the cyst in Case 2 were likely not necessary; we believe that the cyst would have spontaneously resolved after labral repair. Addressing the labral tear can replace the need to disconnect the articular branch to allow cyst resolution and promote neurological recovery. Labral tears may be difficult to identify by preoperative MRI; however, arthrography may be useful in revealing subtle pathology. We believe that suprascapular intraneural ganglia are always associated with labral tears, but certain authors have reported otherwise. ${ }^{35}$ Until now, open surgery has been used to resect intraneural ganglion cysts of the SSN, $, 20,22,24,25,29-31,37$ and if the associated labral tear is not identified or treated, it is imperative to resect the articular nerve branch. However, failure to address the labral tear may result in suboptimal recovery and recurrence. ${ }^{30}$ In summary, the therapeutic objectives in decreasing order of importance are repair of labral pathology, resection of the cystic articular nerve branch, and decompression of the cyst. Cyst decompression, by itself, may be performed before, during, or after surgery to provide symptomatic relief and reduce pressure on the nerve, but it does not address the primary pathol- ogy or prevent recurrence. Our current understanding and surgical approach represents a paradigm shift.

Much of what we have learned about intraneural ganglion cysts has been extrapolated from cases affecting the most common site of occurrence-the common peroneal nerve at the superior tibiofibular joint. The small size of this joint makes arthroscopic exploration difficult. The larger joint cavities associated with shoulder- and hipderived intraneural ganglia offer the opportunity to study the condition from an arthroscopic perspective. General experience in cases at the shoulder confirms the jointrelated pathogenesis of these cysts and the advantages of the arthroscopic approach (i.e., direct, safe treatment of the labral tear and indirect, effective treatment of the intraneural cyst and neuropathy). We uncovered, at the acetabulofemoral joint, a single case of an arthroscopically treated "perineural cyst" of the sciatic nerve near the hip joint. ${ }^{15}$ The authors of that published study believed that the cyst was extraneural, and they simply excised the cyst wall. However, we believe that the cyst was intraneural and that there is likely to have been associated acetabular labral pathology that was not addressed. Likewise, other paralabral cysts at the shoulder region may well be intraneural ganglion cysts, as in our Case 2.

\section{Conclusions}

Rare suprascapular intraneural ganglion cysts have a characteristic clinical and radiological pattern. In keeping with the unifying articular theory, intraneural ganglion cysts share the same clear principles of formation and treatment as the more common paralabral cysts. Learning from current treatment algorithms for paralabral cysts, we 


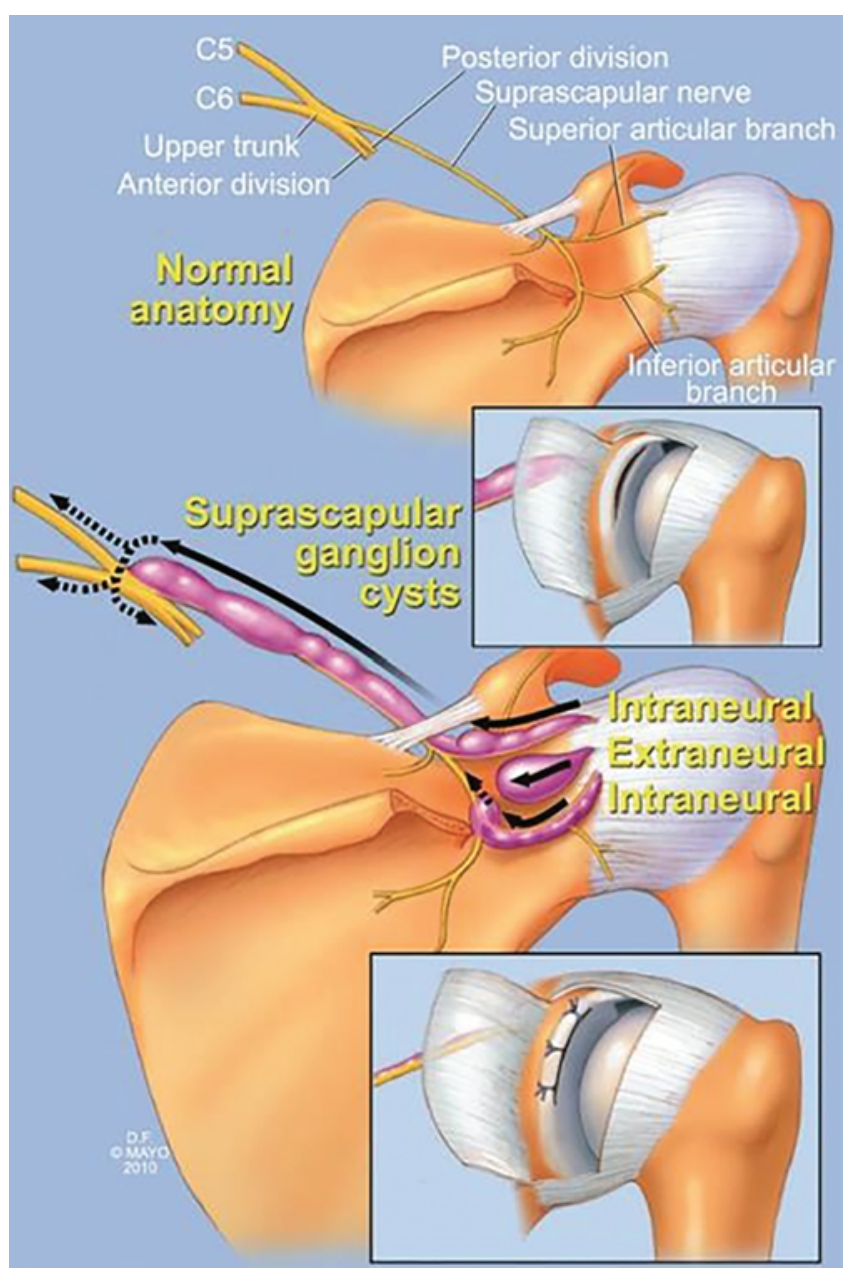

FIG. 8. Anatomical path of the SSN, giving off a superior articular branch to the SSN at the suprascapular notch and an inferior articular branch at the spinoglenoid notch. Ganglion cysts can extend along an extraneural (paralabral) or intraneural (superior and inferior articular branches) route. The only way to prevent recurrence is by repairing the labral tear. Adapted from Spinner et al: J Neurosurg 104:551-557, 2006. Used with permission of the Mayo Foundation for Medical Education and Research. All rights reserved.

believe that intraneural ganglia can be managed without resection of the articular nerve branch or decompression of the cyst, which will gradually involute over time if the causative labral tear is repaired.

\section{Acknowledgments}

We appreciate the assistance of Alexandra P. Wolanskyj, MD, and David Factor of Rochester, Minnesota.

\section{References}

1. Ajmani ML: The cutaneous branch of the human suprascapular nerve. J Anat 185:439-442, 1994

2. Antoniou J, Tae SK, Williams GR, Bird S, Ramsey ML, Iannotti JP: Suprascapular neuropathy. Variability in the diagnosis, treatment, and outcome. Clin Orthop Relat Res (386):131-138, 2001

3. Baums MH, Seil R, Kettler M, Steckel H, Schultz W, Klinger HM: Treatment option in a SLAP-related ganglion cyst re- sulting in suprascapular nerve entrapment. Arch Orthop Trauma Surg 126:621-623, 2006

4. Biedert RM: Atrophy of the infraspinatus muscle caused by a suprascapular ganglion. Clin J Sport Med 6:262-264, 1996

5. Boykin RE, Friedman DJ, Higgins LD, Warner JJ: Suprascapular neuropathy. J Bone Joint Surg Am 92:2348-2364, 2010

6. Chen AL, Ong BC, Rose DJ: Arthroscopic management of spinoglenoid cysts associated with SLAP lesions and suprascapular neuropathy. Arthroscopy 19:E15-E21, 2003

7. Chochole MH, Senker W, Meznik C, Breitenseher MJ: Glenoid-labral cyst entrapping the suprascapular nerve: dissolution after arthroscopic debridement of an extended SLAP lesion. Arthroscopy 13:753-755, 1997

8. Fehrman DA, Orwin JF, Jennings RM: Suprascapular nerve entrapment by ganglion cysts: a report of six cases with arthroscopic findings and review of the literature. Arthroscopy 11:727-734, 1995

9. Ferretti A, Cerullo G, Russo G: Suprascapular neuropathy in volleyball players. J Bone Joint Surg Am 69:260-263, 1987

10. Hadley MN, Sonntag VK, Pittman HW: Suprascapular nerve entrapment. A summary of seven cases. J Neurosurg 64:843-848, 1986

11. Harbaugh KS, Swenson R, Saunders RL: Shoulder numbness in a patient with suprascapular nerve entrapment syndrome: cutaneous branch of the suprascapular nerve: case report. Neurosurgery 47:1452-1456, 2000

12. Hashimoto BE, Hayes AS, Ager JD: Sonographic diagnosis and treatment of ganglion cysts causing suprascapular nerve entrapment. J Ultrasound Med 13:671-674, 1994

13. Ho JC, Iannotti JP: Glenoid labral tear associated paralabral ganglion cyst presenting as a neck mass: a case report. J Shoulder Elbow Surg 19:e10-e13, 2010

14. Horiguchi M: The cutaneous branch of some human suprascapular nerves. J Anat 130:191-195, 1980

15. Hwang DS, Kang C, Lee JB, Cha SM, Yeon KW: Arthroscopic treatment of piriformis syndrome by perineural cyst on the sciatic nerve: a case report. Knee Surg Sports Traumatol Arthrose 18:681-684, 2010

16. Iannotti JP, Ramsey ML: Arthroscopic decompression of a ganglion cyst causing suprascapular nerve compression. Arthroscopy 12:739-745, 1996

17. Kessler MA, Stoffel K, Oswald A, Stutz G, Gaechter A: The SLAP lesion as a reason for glenolabral cysts: a report of five cases and review of the literature. Arch Orthop Trauma Surg 127:287-292, 2007

18. Lichtenberg S, Magosch P, Habermeyer P: Compression of the suprascapular nerve by a ganglion cyst of the spinoglenoid notch: the arthroscopic solution. Knee Surg Sports Traumatol Arthrosc 12:72-79, 2004

19. Mallon WJ: Editor's note. J Shoulder Elbow Surg 19:633634, 2010

20. Mizuno K, Usui Y, Harada Y, Hirohata K: Infraspinatus muscle paralysis due to epineural ganglion of the suprascapular nerve. Jpn J Rheum Joint Surg 9:521-526, 1990

21. Moore TP, Fritts HM, Quick DC, Buss DD: Suprascapular nerve entrapment caused by supraglenoid cyst compression. J Shoulder Elbow Surg 6:455-462, 1997

22. Nägele M, Lienemann A, Hahn D, Witt T: [Intraneuronal ganglia: value of diagnostic radiologic possibilities.] Digitale Bilddiagn 8:14-17, 1988 (Ger)

23. Piatt BE, Hawkins RJ, Fritz RC, Ho CP, Wolf E, Schickendantz M: Clinical evaluation and treatment of spinoglenoid notch ganglion cysts. J Shoulder Elbow Surg 11:600-604, 2002

24. Rezzouk J, Durandeau A: [Nerve compression by mucoid pseudocysts: arguments favoring an articular cause in 23 patients.] Rev Chir Orthop Reparatrice Appar Mot 90:143-146, 2004 (Fr) 
25. Sanger J, Cortes W, Yan JG: Intraneural ganglion of the suprascapular nerve: case report. J Hand Surg Am 31:40-44, 2006

26. Schroder CP, Skare O, Stiris M, Gjengedal E, Uppheim G, Brox JI: Treatment of labral tears with associated spinoglenoid cysts without cyst decompression. J Bone Joint Surg Am 90:523-530, 2008

27. Shon MS, Jung SW, Kim JW, Yoo JC: Arthroscopic all-intraarticular decompression and labral repair of paralabral cyst in the shoulder. J Shoulder Elbow Surg 24:e7-e14, 2015

28. Skirving AP, Kozak TK, Davis SJ: Infraspinatus paralysis due to spinoglenoid notch ganglion. J Bone Joint Surg Br 76:588-591, 1994

29. Spinner RJ, Amrami KK: Intraneural ganglion of the suprascapular nerve: Case report. J Hand Surg Am 31:1698-1699, 2006

30. Spinner RJ, Amrami KK, Kliot M, Johnston SP, Casañas J: Suprascapular intraneural ganglia and glenohumeral joint connections. J Neurosurg 104:551-557, 2006

31. Spinner RJ, Amrami KK, Wang H, Kliot M, Carmichael SW: Cross-over: a generalizable phenomenon necessary for secondary intraneural ganglion cyst formation. Clin Anat 21:111-118, 2008

32. Spinner RJ, Atkinson JL, Tiel RL: Peroneal intraneural ganglia: the importance of the articular branch. A unifying theory. J Neurosurg 99:330-343, 2003

33. Spinner RJ, Hébert-Blouin MN, Dahm DL, Amrami KK: Two different pathways for suprascapular intraneural ganglion cysts along two distinct articular branches from the glenohumeral joint. Clin Anat 23:462-465, 2010

34. Spinner RJ, Scheithauer BW, Amrami KK: The unifying articular (synovial) origin of intraneural ganglia: evolutionrevelation-revolution. Neurosurgery 65 (4 Suppl):A115A124, 2009

35. Sureka J, Panwar S: MR imaging of suprascapular neuropathies. J Musculoskelet Res 15:1230003, 2012

36. Tirman PF, Feller JF, Janzen DL, Peterfy CG, Bergman AG: Association of glenoid labral cysts with labral tears and glenohumeral instability: radiologic findings and clinical significance. Radiology 190:653-658, 1994
37. Usui Y, Harada Y, Mizuno K, Hirohata K: Infraspinatus paralysis by a suspected intraneural ganglion. A case report. Rinnshouseikei 20:887-891, 1985

38. Vorster W, Lange CP, Briët RJ, Labuschagne BC, du Toit DF, Muller CJ, et al: The sensory branch distribution of the suprascapular nerve: an anatomic study. J Shoulder Elbow Surg 17:500-502, 2008

39. Westerheide KJ, Dopirak RM, Karzel RP, Snyder SJ: Suprascapular nerve palsy secondary to spinoglenoid cysts: results of arthroscopic treatment. Arthroscopy 22:721-727, 2006

40. Youm T, Matthews PV, El Attrache NS: Treatment of patients with spinoglenoid cysts associated with superior labral tears without cyst aspiration, debridement, or excision. Arthroscopy 22:548-552, 2006

41. Zehetgruber H, Noske H, Lang T, Wurnig C: Suprascapular nerve entrapment. A meta-analysis. Int Orthop 26:339-343, 2002

\section{Disclosure}

Dr. Smith owns stock in Sonex Health and Tenex Health; holds a patent with Tenex Health; holds a license agreement with Tenex Health; and is the chief medical officer of Sonex Health. Dr. Iannotti holds a patent with the Cleveland Clinic and DePuy Synthes.

\section{Author Contributions}

Conception and design: Spinner, Smith, Dahm. Acquisition of data: Spinner, Smith, Howe, Iannotti, Dahm. Analysis and interpretation of data: Spinner, Prasad, Howe, Amrami, Dahm. Drafting the article: all authors. Critically revising the article: Spinner, Prasad, Smith, Dahm. Reviewed submitted version of manuscript: Spinner, Prasad. Study supervision: Spinner.

\section{Correspondence}

Robert J. Spinner, Mayo Clinic, Gonda 8-214S, Rochester, MN 55905. email: spinner.robert@mayo.edu. 